\title{
Application of Modern Scanning/Transmission Electron Microscope with Pixelated STEM Detector for Radiation Damage Study
}

Joven Lim ${ }^{1}$, Eric Prestat ${ }^{2}$, Anna Carlsson ${ }^{3}$, Graeme Greaves ${ }^{4}$, Stephen Donnelly ${ }^{4}$, Quentin Ramasse ${ }^{5}$ and M. Grace Burke ${ }^{6}$

${ }^{1}$ UK Atomic Energy Authority, Abingdon, England, United Kingdom, ${ }^{2}$ University of Manchester and SuperSTEM Laboratory, Manchester, England, United Kingdom, ${ }^{3}$ Thermo Fisher Scientific, Eindhoven, Noord-Brabant, Netherlands, ${ }^{4}$ University of Huddersfield, Huddersfield, England, United Kingdom, ${ }^{5}$ SuperSTEM Laboratory and University of Leeds, Daresbury, England, United Kingdom, ${ }^{6}$ University of Manchester, Manchester, Lancashire, England, United Kingdom

Conventional Transmission Electron Microscopy (CTEM) was the first characterisation method used to visualise and understand radiation-induced damage, especially dislocation loops, that causes the properties degradation in metal for nuclear application. Weak Beam Dark Field (WBDF) imaging technique that widely used to characterise small dislocation loops, also known as black dots, is an effective technique to determine the type and nature of irradiation-induced dislocation loops via the classical $g . b$ method. However, the collection of a complete set of WBDF data from an irradiated sample can be time consuming as it requires to cover multiple $g$-vectors in order to carry out the $g$.b elimination process. Moreover, the area of interest will expose to a large electron dose. This might be a problem for some materials as some of irradiation-induced dislocation loops are sensitive to local electron beam heating/ high beam current.

AtomCRaD, an electron microscopy (EM) technique development research project that funded by EUROfusion, has investigated new methodology to characterise radiation damage using latest hardware and software technologies available in the field of EM. In this project, we have investigated the effectiveness of using pixelated STEM detector to study irradiation-induced dislocation loops in ion irradiated tungsten. Figure 1 shows the reconstructed Bright Field (BF), Dark field (DF) and Annular Dark Field (ADF) images from a collected a 4D-STEM data set. The reconstructed images were compared with those that collected via conventional detector under the same diffraction conditions. The comparisons confirmed the quality of the reconstructed images are as good as those collected via conventional detector. One of the main advantages using 4D-STEM data for radiation damage analysis is that DF of different $g$ vectors and $\mathrm{ADF}$ images with different collection angles can be reconstructed. The effects of convergence angle and the $g . b$ analysis performed using 4D-STEM data will be discussed in detailed [1,2]. 

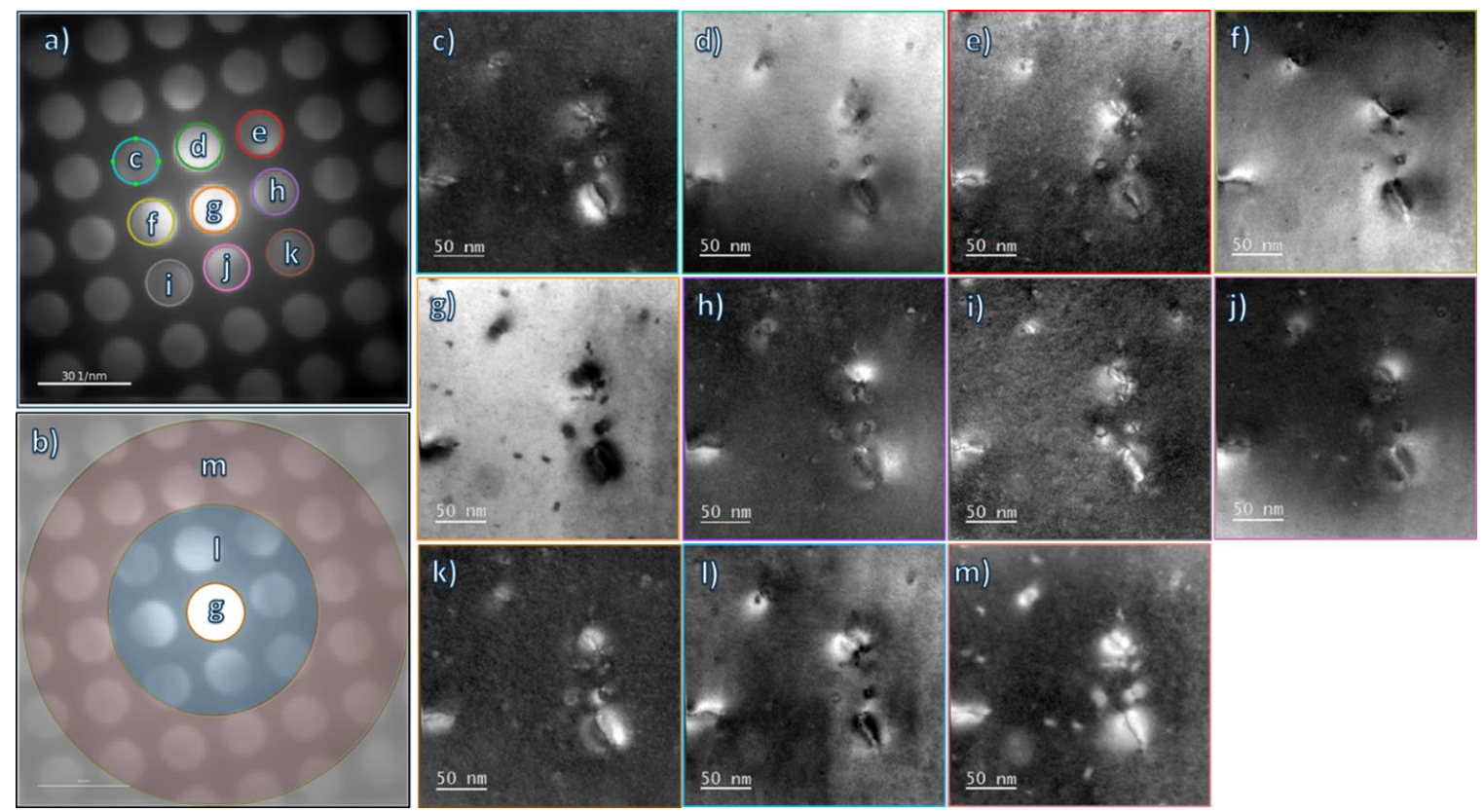

Figure 1. Reconstructed Bright Field, Dark Field and Annular Dark Field images generated using virtual apertures from a 4D-STEM data set that was collected from $150 \mathrm{keV}$ self-ion irradiate pure W $(99.95 \%)$ up to $0.005 \mathrm{dpa}$.

\section{References}

[1] The sample preparation equipment used UKAEA's Materials Research Facility, which has been funded by and is part of the UK's National Nuclear User Facility and Henry Royce Institute for Advanced Materials [Grant No. EP/P021727/1]

[2] This work has been carried out within the framework of the EUROfusion Consortium and has received funding from the Euratom research and training programme 2014-2018 and 2019-2020 under grant agreement No 633053 and from RCUK [grant number EP/T012250/1]. To obtain further information on the data and models underlying this paper please contact PublicationsManager@ukaea.uk*. The views and opinions expressed herein do not necessarily reflect those of the European Commission. 